\title{
Influence of antenatal glucocorticoid on preterm lamb diaphragm
}

\author{
Tanzila Mahzabin ${ }^{1,2}$, J. Jane Pillow ${ }^{1,2}$, Gavin J. Pinniger ${ }^{1}$, Anthony J. Bakker ${ }^{1}$, Peter B. Noble ${ }^{1,2}$, Robert B. White ${ }^{1}$, \\ Kanakeswary Karisnan ${ }^{1,2,3}$ and Yong Song ${ }^{1,2,4,5}$
}

BACKGROUND: Pregnant women at a high risk of preterm delivery receive glucocorticoids to accelerate fetal lung maturation and surfactant synthesis. However, the effect of antenatal steroids on the developing diaphragm remains unclear. We hypothesized that maternal betamethasone impairs the fetal diaphragm, and the magnitude of the detrimental effect increases with longer duration of exposure. We aimed to determine how different durations of fetal exposure to maternal betamethasone treatment influence the fetal diaphragm at the functional and molecular levels.

METHODS: Date-mated merino ewes received intramuscular injections of saline (control) or two doses of betamethasone $(5.7 \mathrm{mg}$ ) at an interval of $24 \mathrm{~h}$ commencing either 2 or 14 days before delivery. Preterm lambs were killed after cesarean delivery at 121-day gestational age. In vitro contractile measurements were performed on the right hemidiaphragm, whereas molecular/cellular analyses used the left costal diaphragm.

RESULTS: Different durations of fetal exposure to maternal betamethasone had no consistent effect on the protein metabolic pathway, expression of glucocorticoid receptor and its target genes, cellular oxidative status, or contractile properties of the fetal lamb diaphragm.

CONCLUSION: These data suggest that the potential benefits of betamethasone exposure on preterm respiratory function are not compromised by impaired diaphragm function after low-dose maternal intramuscular glucocorticoid exposure.

A functional respiratory system is critical to successfully transit from intrauterine fetal breathing to extrauterine spontaneous breathing at birth. Preterm babies are born before their vital organs are sufficiently matured to support extrauterine life. Not surprisingly, the incidence of respiratory failure is higher in preterm infants than in any other time of life. Antenatal betamethasone is routinely administered to women at high risk of preterm delivery to minimize neonatal respiratory failure via the acceleration of fetal lung maturation. More than $85 \%$ of infants born preterm $(<34$ weeks) are exposed to glucocorticoids antenatally $(1,2)$. Other widely reported beneficial effects of antenatal corticosteroids in preterm neonates include improved surfactant production and reductions in cerebroventricular hemorrhage, necrotizing enterocolitis, the need for respiratory support, infectious morbidity, and neonatal death (2). Conversely, the high potency and potential long-term adverse effects of glucocorticoid exposure on organ development are driving research to minimize effective glucocorticoid dose (3).

The effect of antenatal glucocorticoids on the developing respiratory muscles remains unclear. The premature diaphragm is structurally and functionally immature and characterized by a predominant fast-twitch myosin heavychain (MHC) composition (4,5), reduced contractile force production (6), reduced antioxidant capacity (7), and inefficient calcium $\left(\mathrm{Ca}^{2+}\right)$ handling properties (8). Increased mechanical load imposed by a highly compliant chest wall, low levels of endogenous surfactant, and noncompliant, structurally immature, lungs present further challenges for the preterm diaphragm. If maternal steroid exposure has detrimental effects on the already-compromised preterm diaphragm, this may cause severe diaphragm dysfunction and may increase the risk of respiratory failure.

In adults, glucocorticoid treatment causes significant skeletal muscle myopathy featuring contractile impairment and atrophy. Mechanistic understanding of glucocorticoidinduced myopathy is derived predominantly from adult rodent studies. The extent of adult muscle weakness depends on the dose, duration, and type of steroid treatment (9). Glucocorticoid-induced skeletal muscle atrophy is characterized by depressed protein synthesis and enhanced proteolysis (10-12). Atrophy reduces muscle mass and consequently force production, which is associated with impaired diaphragm function in adult rats (13). The catabolic action of glucocorticoid involves glucocorticoid receptor (GR) activation along with a wide range of signaling mediators and local growth factors (14). In addition, glucocorticoids significantly increase the expression of stress- and inflammation-related

${ }^{1}$ School of Human Sciences, The University of Western Australia, Perth, Western Australia, Australia; ${ }^{2}$ Centre for Neonatal Research and Education, Division of Paediatrics and Child Health, Medical School, The University of Western Australia, Perth, Western Australia, Australia; ${ }^{3}$ School of Pharmacy, International Medical University, Kuala Lumpur, Malaysia; ${ }^{4}$ School of Public Health, Curtin University, Perth, Western Australia, Australia; ${ }^{5}$ Centre for Genetic Origins of Health and Disease, The University of Western Australia and Curtin University, Perth, Western Australia, Australia. Correspondence: Yong Song (yong.song1@curtin.edu.au)

Received 11 September 2016; accepted 30 March 2017; advance online publication 17 May 2017. doi:10.1038/pr.2017.99 


\section{Articles | Mahzabin et al.}

genes in healthy adult human skeletal muscles (15). Thus, the complex mechanisms underlying glucocorticoid-induced muscle weakness involve the regulation of metabolic pathways and gene transcription, and they are studied extensively in adult skeletal muscle.

Contrary to the adult diaphragm, the effect of glucocorticoids on the preterm diaphragm is unclear. High-dose $(0.5 \mathrm{mg} / \mathrm{kg})$ maternal intramuscular (IM) betamethasone given 7 and 3 days before birth impedes postnatal (21 days) diaphragm contractile function in rat pups (16). Two low-dose $(0.17 \mathrm{mg} / \mathrm{kg})$ IM betamethasone doses given to pregnant baboons at a $24 \mathrm{~h}$ interval commencing 2 days prior to delivery decreased protein synthesis signaling in preterm but not in term skeletal muscle (vastus lateralis), suggesting gestational age sensitivity to adverse consequences of glucocorticoid exposure (17). In contrast, maternal IM dexamethasone $(12 \mathrm{mg})$ had no effect on the anabolic signaling pathway of fetal skeletal muscle (18). Thus, there is evidence of impaired preterm diaphragm muscle function in response to high-dose maternal steroid and conflicting impact of antenatal glucocorticoids on other skeletal muscles, and an unclear impact of glucocorticoid dose on fetal skeletal muscle function.

The current study aimed to determine the effect of different durations of antenatal betamethasone exposure on contractile function and muscle atrophy in the preterm fetal lamb diaphragm using a low-dose maternal glucocorticoid treatment. We hypothesized that low-dose antenatal betamethasone would impair the immature diaphragm by redirecting the protein homeokinetic process toward net protein catabolism, thus contributing to muscular atrophy and increasing susceptibility to fatigue and dysfunction. We also hypothesized that fetal diaphragm atrophy and contractile dysfunction would be more evident in lambs exposed to maternal betamethasone at an earlier stage in development, where it could potentially exert a greater net catabolic impact on diaphragm development.

\section{METHODS}

\section{Experimental Design}

All the experiments were approved by the University of Western Australia Animal Ethics Committee (RA/3/400/1023). Date-mated Merino ewes were randomly divided into three experimental groups. The control group $(n=6)$ received IM saline injections and two experimental groups received two IM injections of $5.7 \mathrm{mg}(\sim 0.1 \mathrm{mg} /$ $\mathrm{kg} \times$ two doses $=0.2 \mathrm{mg} / \mathrm{kg}$ total exposure) betamethasone (Celestone from Shering Plough, Kenilworth, NJ) at an interval of $24 \mathrm{~h}$ at either 2 days $(n=7)$ or 14 days $(n=8)$ prior to delivery. Two different time points were selected to observe the effect of acute short exposure ( 2 days) and long duration exposure (14 days) of the fetus to maternal betamethasone treatment. Preterm lambs delivered at 121day gestational age via cesarean section were killed immediately with pentobarbitone $(150 \mathrm{mg} / \mathrm{kg}$, intravenous; Pitman-Moore, Sydney, NSW, Australia). The right hemidiaphragm was removed for in vitro contractile measurements and the left costal hemidiaphragm was snap-frozen in liquid nitrogen for molecular and biochemical analyses.

\section{RNA Isolation for Quantitative Reverse-Transcriptase PCR}

The left costal hemidiaphragm (30 mg tissue) was homogenized and used for total RNA purification using the RNeasy Mini kit with an on-column DNase treatment (Qiagen Pty, Melbourne, Victoria, Australia). Isolated RNA (1000 ng) was reverse-transcribed into complementary DNA in a $20 \mu \mathrm{l}$ reaction using the QuantiTect Reverse Transcription Kit (Qiagen Pty). Real-time amplification reactions were conducted using the Rotor-Gene SYBR Green PCR Kit (Qiagen Pty) on a Rotor-gene 6000 real-time PCR system (Corbett Life Science, Mortlake, Victoria, Australia), under the following cycling conditions: $5 \mathrm{~min}$ at $95^{\circ} \mathrm{C}, 40$ cycles of $3 \mathrm{~s}$ at $95^{\circ} \mathrm{C}$, $20 \mathrm{~s}$ at $60^{\circ} \mathrm{C}$, and $20 \mathrm{~s}$ at $72{ }^{\circ} \mathrm{C}$. Gene expression level was assessed for atrophy-related genes, muscle ring-finger protein 1 (MuRF1) and muscle atrophy F-box (Atrogin-1/MAFbx), GR $\alpha$, and five of its direct target genes as follows: insulin-like growth factor 1 (IGF1), insulin receptor substrate 1(IRS1), phosphoinositide-3 kinase regulatory subunit $1(p 85 \alpha)$, Krüppel-like factor 15 (klf15), interleukin-10 (IL-10), MHC isoforms (MHC neonatal, I, IIa, and IIx), antioxidant genes (Catalase; GPX1, glutathione peroxidase 1, and SOD1, superoxide dismutase 1). Primers were designed on ovine-specific mRNA sequence, as described previously $(6,7)$, and novel primers are listed in Table 1. The expression levels of genes of interest were normalized against the geometrical mean of $18 S \mathrm{RNA}, \mathrm{GAPDH}$, and

Table 1. Novel primer sequences designed for real-time PCR

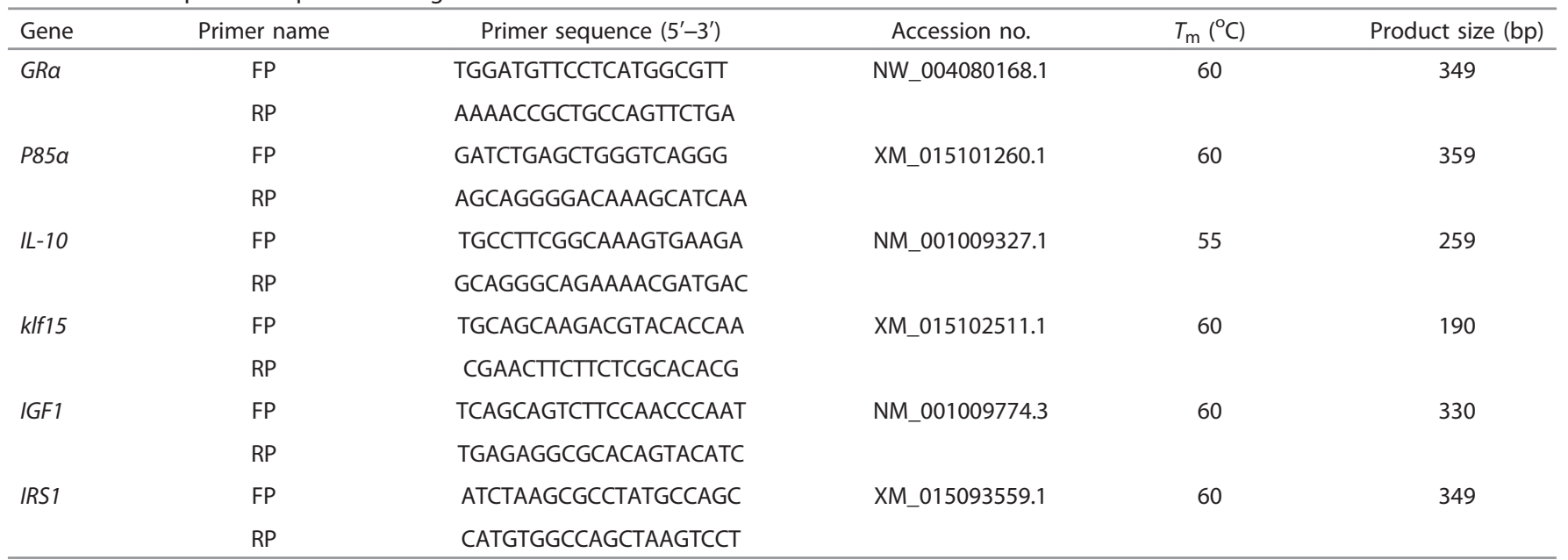

FP, forward primer; GRa, glucocorticoid receptor alpha; IGF1, insulin-like growth factor 1; IL-10, interleukin-10; IRS1, insulin receptor substrate 1; kIf15, Krüppel-like factor 15; P85a, phosphoinositide-3 kinase regulatory subunit 1; RP, reverse primer. 


\section{Maternal steroid and preterm diaphragm Articles}

$\beta$-actin genes. The $2^{-\Delta \Delta C T}$ method (19) was used to calculate change in gene expression relative to the control group.

\section{Protein Fractionation for Western Blot}

Cytosolic and nuclear protein fractions were prepared from $30 \mathrm{mg}$ diaphragm tissue using the NE-PER Nuclear and Cytoplasmic Extraction Kit with the inclusion of protease inhibitor cocktail (Thermo Scientific, Billerica, MA). Protein concentration was measured for cytoplasmic and nuclear fractions using the Bradford method (Sigma, Sydney, New South Wales, Australia). Protein samples $(10 \mu \mathrm{g})$ were resolved on $4-15 \%$ TGX stain-free gels (BioRad, Gladesville, New South Wales, Australia) and were transferred onto nitrocellulose membrane using a Trans Turbo Blot system (BioRad). After blocking with phosphate-buffered saline containing 5\% skimmed milk, the membranes were incubated at $4{ }^{\circ} \mathrm{C}$ overnight with primary antibodies (Cell Signaling Technology, Carlsbad, CA) of protein synthesis signaling components at a dilution of $1: 1,000$ (mTOR; ribosomal protein s-6 kinase beta1, p70S6K) or 1:2,000 (protein kinase B, Akt; translation initiation factors 4e-binding protein 1,4EBP1). Bound antibodies were detected with an antirabbit immunoglobulin conjugated with horseradish peroxidase at 1:3,000 dilution (Cell Signaling Technology) for $1 \mathrm{~h}$ at room temperature. The blots were developed by adding a SuperSignal West Pico Chemiluminescent Substrate (Thermo Scientific). Immunoreactive protein signals were quantified using a ChemiDoc MP Imaging system (Bio-Rad). The values for phosphorylated protein were normalized to total protein content of the same blot.

\section{Biochemical Assays of Proteolysis and Oxidative Stress}

The total protein was extracted from $30 \mathrm{mg}$ diaphragm tissue, and protein concentration was measured as described previously (7). The total protein content was used for assessing $20 \mathrm{~S}$ proteasome levels (major component of ubiquitine proteasome pathway, UPP) and protein oxidation status. Chymotrypsin-like protease activity in diaphragm samples was measured using a fluorogenic kit (BML-AK 740 assay kit, Enzo Life Sciences, Farmingdale, NY) and was normalized against total protein concentration. The protein carbonyl content (an indicator of cellular oxidation status) was measured using a commercially available colorimetric kit (Protein Carbonyl Colorimetric assay kit, Cayman, Ann Arbor, MI).

\section{Diaphragm Contractile Properties}

Contractile properties of diaphragm muscle were measured as described previously (6). Briefly, longitudinal strips of diaphragm muscle fibers were mounted in an in vitro muscle test system (model 1205, Aurora Scientific, Aurora, Ontario, Canada) containing Kreb's physiological salt solution (in $\mathrm{mM}: \mathrm{NaCl}, 109 ; \mathrm{KCl}, 5 ; \mathrm{MgCl}_{2}, 1$; $\mathrm{CaCl}_{2}, 4 ; \mathrm{NaHCO}_{3}, 24 ; \mathrm{NaH}_{2} \mathrm{PO}_{4}, 1$; sodium pyruvate, 10). The Kreb's solution was bubbled with carbogen $\left(98 \% \mathrm{O}_{2}, 5 \% \mathrm{CO}_{2}\right)$ to maintain $\mathrm{pH}$ approximately at 7.4. The contractile parameters measured include maximum isometric tetanic force $\left(P_{\mathrm{o}}\right)$, peak twitch force $\left(P_{\mathrm{t}}\right)$, time to peak (TTP), half relaxation time $(1 / 2 \mathrm{RT})$, and maximum rate of force development $(\mathrm{d} F / \mathrm{d} T)$ of twitch contractions. The isometric force-frequency relationship was determined for stimulation frequencies of $1-80 \mathrm{~Hz}$. Susceptibility to fatigue was evaluated from a series of 150 maximum isometric tetanic contractions. The fatigue index was determined from the ratio of the force produced during the 150th contraction relative to the 1st contraction (20), in which a higher number indicates a greater fatigue resistance. The optimal muscle length $\left(L_{\mathrm{o}}\right)$ and mass of the diaphragm strip were recorded at the end of the experiment and were used to estimate cross-sectional area of the diaphragm strip, assuming a muscle density of $1.056 \mathrm{~g} / \mathrm{cm}^{3}$. To account for slight differences in the size of the dissected diaphragm strips, the absolute force was normalized to cross-sectional area and expressed as specific force $\left(\mathrm{N} / \mathrm{cm}^{2}\right)$.
Table 2. Fetal lamb characteristics

\begin{tabular}{lccc}
\hline & Control & 2-day Beta & 14-day Beta \\
\hline$n$ & 6 & 7 & 8 \\
Body weight $(\mathrm{kg})$ & $2.19 \pm 0.35$ & $2.13 \pm 0.27$ & $2.18 \pm 0.33$ \\
Diaphragm weight (g) & $2.19 \pm 0.58$ & $2.25 \pm 0.52$ & $2.01 \pm 0.61^{\mathrm{a}}$ \\
$\begin{array}{l}\text { Relative diaphragm } \\
\text { weight (\%) }\end{array}$ & $0.099 \pm 0.012$ & $0.100 \pm 0.014$ & $0.080 \pm 0.035^{\mathrm{a}}$ \\
$\begin{array}{l}\text { Sex ratio (male:female) } \\
\text { Pregnancy ratio }\end{array}$ & $3: 3$ & $5: 2$ & $5: 3$ \\
(singleton:twin) & $4: 2$ & $5: 2$ & $3: 5$ \\
\hline
\end{tabular}

Beta, betamethasone; Relative diaphragm weight, diaphragm weight/body weight Values are mean \pm standard SD.

${ }^{a} n=7$ only for the diaphragm weight.

\section{Data Analysis}

Data are presented as mean (SD) or median (quartiles) using box and whisker plots showing all data points minimum to maximum. Graph Pad PRISM (version 6.07, GraphPad Software,, San Diego, CA) was used for statistical analysis. Normally distributed data were analyzed using one-way analysis of varinace or unpaired $t$-tests as appropriate. For non-normally distributed data, differences between groups (control, 2-day betamethasone, and 14-day betamethasone) were assessed using the Kruskal-Wallis multiple comparison test or MannWhitney $U$-test. $P<0.05$ was considered statistically significant.

\section{RESULTS}

\section{Lamb Characteristics}

Pregnant Merino sheep in this study weighed between 55 and $85 \mathrm{~kg}$. There were no significant differences in body weight or diaphragm weight between treatment and control groups (Table 2). The fetal sex distributions and whether fetuses were from singleton or multiple pregnancies are indicated in Table 2.

\section{Proteolytic Signaling Pathway}

Expression levels (messenger RNA (mRNA)) from MuRF1 and $M A F b x$ (atrophy genes) did not change significantly after treatment with antenatal betamethasone (Figure 1a,b). The ubiquitin proteasome activity also remained unaffected, as determined by the absence of significant differences in the $20 \mathrm{~S}$ proteasome activity in the diaphragm samples (Figure 1c).

\section{Anabolic Signaling Pathway}

Protein activity was evaluated for four intracellular mediators of anabolic signaling (Akt, mammalian target of rapamycin (mTOR), 4EBP1, and p70S6k). Protein activity was determined as the ratio of phosphorylated (active) over the total protein $(\mathrm{P} / \mathrm{T})$ content. There were no significant differences in the protein activity of any of the examined signaling components in 2-day treatment, 14-day treatment, and control groups $(P>0.05$, Figure 2$)$.

\section{GR and its Direct Target Gene Regulation}

Gene expression (mRNA) of $G R \alpha$ in the fetal diaphragm of the 2- and 14-day betamethasone groups did not differ significantly from that in the control group (Figure 3a). mRNA levels of GR $\alpha$ target genes, IGF1, IRS1, $p 85 \alpha, k l f 15$, 


\section{Articles | Mahzabin et al.}
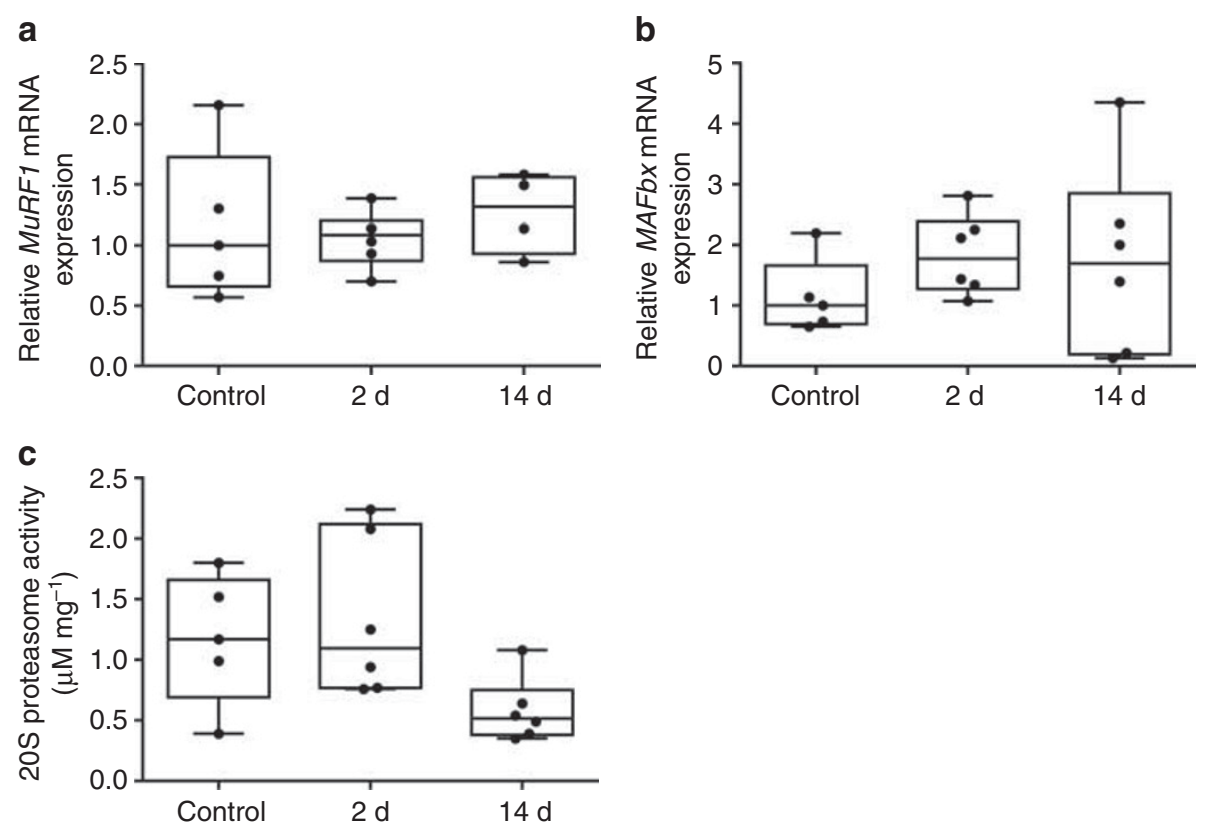

Figure 1. Proteolytic signaling. MuRF1 (a) and MAFbx (b) mRNA levels and 20S proteasome activity (c) in diaphragm samples from control $(n=5)$ and maternal betamethasone treatment groups of 2 days $(n=6)$ and 14 days ( $n=6$, except $n=4$ for MuRF1 quantitative reverse-transcriptase PCR (RTqP(R)). Values are expressed relative to median of the control group for RT-qPCR analysis. Arbitrary fluorescent units from $20 \mathrm{~S}$ proteasome assay are normalized against total protein concentration of respective diaphragm samples.

and $I L-10$, also remained unchanged when compared with controls after a 2- and 14-day low-dose antenatal betamethasone exposure (Figure $3 \mathbf{b}-\mathbf{f}$ ).

\section{Oxidative Status}

Gene expression (mRNA) was determined for three antioxidant genes (Catalase, GPX1, and SOD1). Catalase or GPX1 expression did not change across different tested groups (Figure 4a,b). However, SOD1 mRNA level was significantly upregulated (Kruskal-Wallis multiple comparison; control vs. the 2-day exposure group, $P=0.001)$ after a 2-day betamethasone exposure compared with control. The increased expression of SOD1 did not persist after a 14-day betamethasone exposure (Figure 4c). Protein carbonyl content remained unaltered in maternal glucocorticoid-treated groups compared with controls (Figure 4d).

\section{MHC Gene Expression}

MHC I and MHC neonatal gene expression did not differ between treatment and control groups (Figure 5a,b). However, MHC IIa gene expression was upregulated after the 2-day betamethasone exposure compared with control and the 14-day exposure groups (Figure 5c). The increase in MHC IIx expression level 2 days after betamethasone was significant only when compared with the 14-day betamethasone exposure (Figure $\mathbf{5 d}$ ).

\section{Contractile Properties}

Contractile measurements, including maximum tetanic force, peak twitch force, time to peak twitch force, half relaxation time, maximum rate of force development, and fatigue index, were not significantly different in the fetal lamb diaphragm exposed to maternal betamethasone treatment compared with controls (Table 3). The force-frequency data were normalized to the maximum isometric force and are presented in Figure 6. Two-day betamethasone exposure produced a small but significant decrease in normalized force at stimulation frequencies of 1 and $5 \mathrm{~Hz}$ compared with controls $(P<0.05)$. The force-frequency relationship in the 14-day betamethasone group was similar to controls.

\section{DISCUSSION}

We investigated cellular, molecular, and functional properties of the diaphragm in a preterm fetal lamb model to elucidate the effect of antenatal steroid exposure on the preterm diaphragm. Our experimental observations do not support our hypothesis that antenatal steroid exposure would have significant detrimental effects on the integrity of the preterm diaphragm. Moreover, different durations of exposure to maternal antenatal glucocorticoid did not have any differential or consistent influence on the fetal lamb diaphragm.

The maternal glucocorticoid dosage used in this study (two doses of $5.7 \mathrm{mg}$ per dose betamethasone IM given at $24 \mathrm{~h}$ intervals) is $50 \%$ of the dose used for routine human clinical practice (two doses of $11.4 \mathrm{mg}$ betamethasone IM at $24 \mathrm{~h}$ interval). Dosing regimens used in pregnant women originate from studies in sheep and have remained unchanged for over 40 years since the original randomized clinical trial (21). Nonetheless, two $11.4 \mathrm{mg}$ betamethasone doses frequently 
a

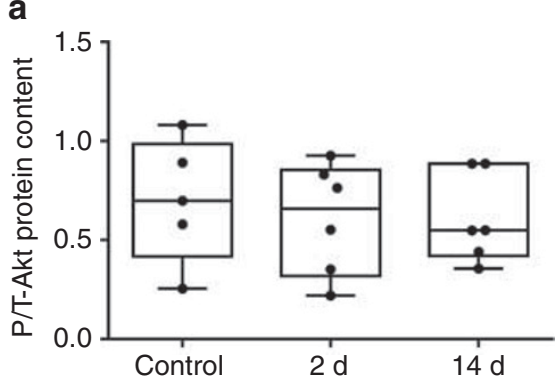

C

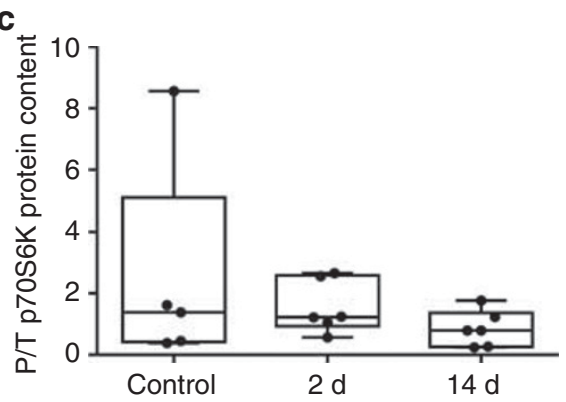

b

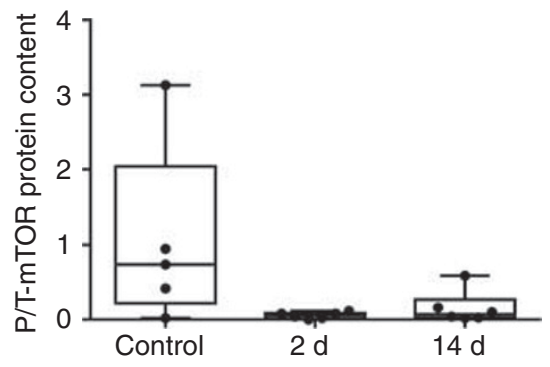

d

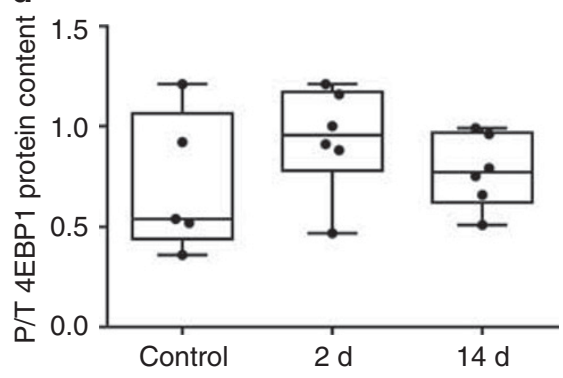

Figure 2. Anabolic signaling. Ratio of phosphorylated/total protein content (P/T) of four components of anabolic signaling in diaphragm samples from control $(n=5)$, and 2-day $(n=6)$ and 14-day $(n=6)$ betamethasone exposure groups for Akt (a), mTOR (b), 4EBP1 (c), and p70S6K (d). Box and whisker plots represent median, minimum, and maximum values.
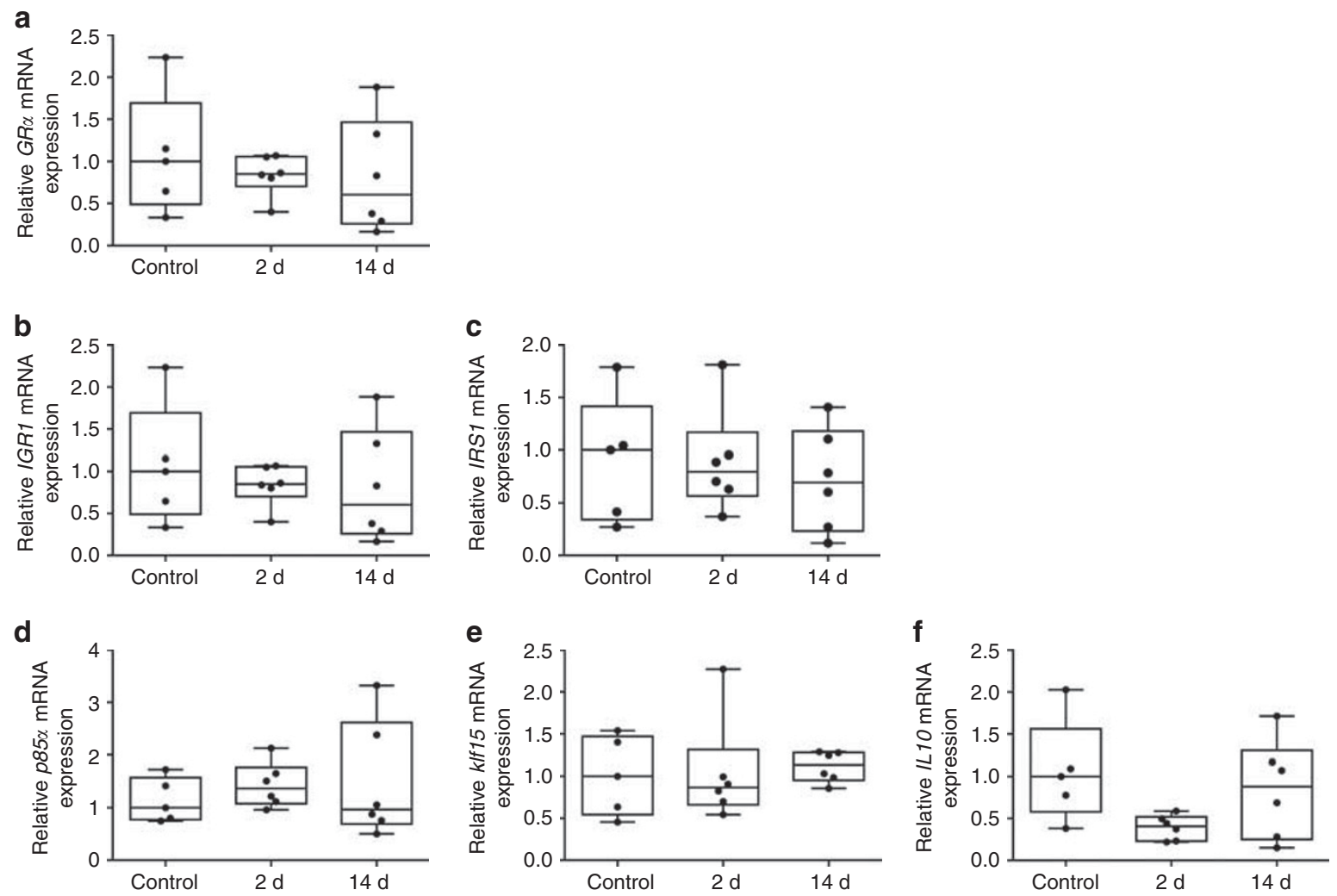

Figure 3. Expression levels of glucocorticoid receptor and downstream target genes. The relative mRNA expression for GRa (a), its downregulated direct target genes IGF1 (b) and IRS1 (c), and upregulated direct target genes p85a (d), kIf15 (e), and IL-10 (f) in the fetal diaphragm muscle from control $(n=5), 2$-day $(n=6)$, and 14-day $(n=6)$ betamethasone-exposed animals. 

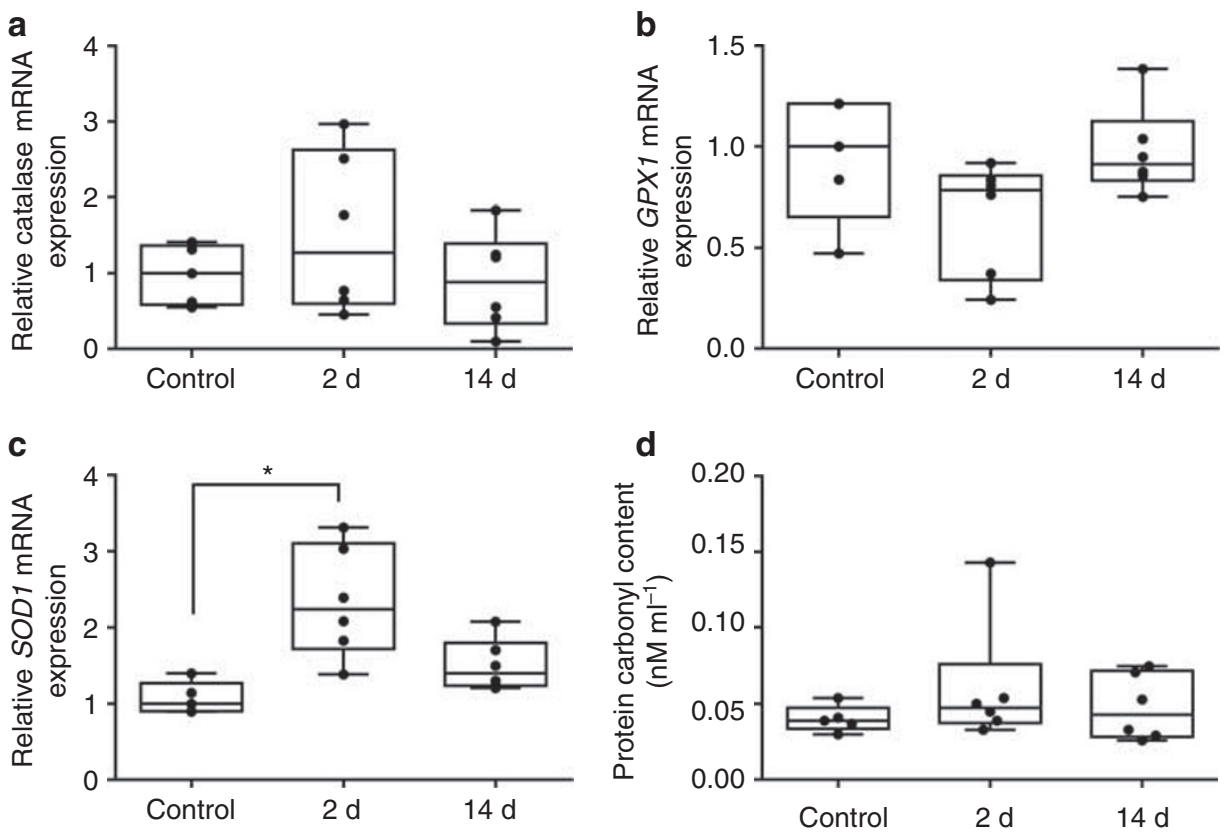

Figure 4. Oxidation status. The relative mRNA expression for antioxidant genes Catalase (a), GPX1 (b), SOD1 (c), and protein carbonyl content (d) in the preterm diaphragm from control $(n=5), 2$-day $(n=6)$, and 14-day $(n=6)$ betamethasone exposure groups. Data are expressed relative to median of control group. ${ }^{*} P<0.05$.
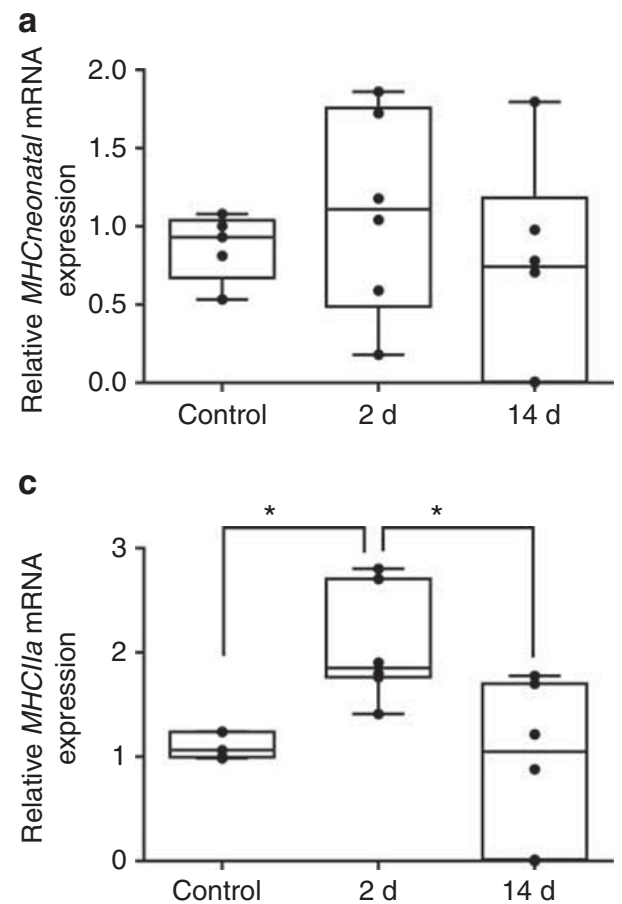

b

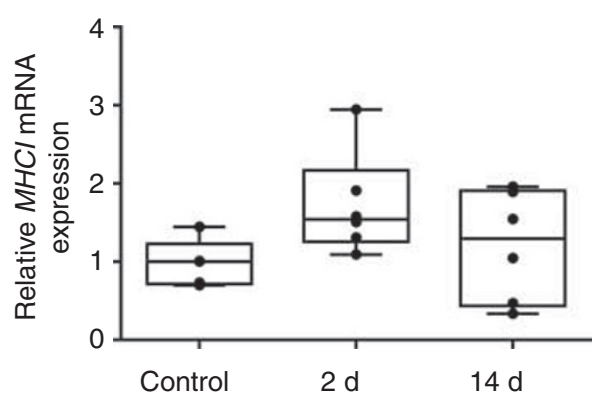

d

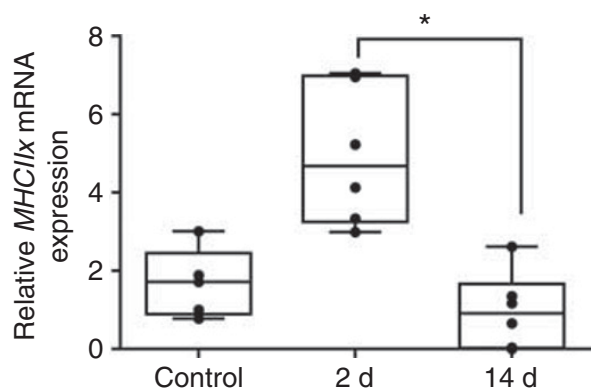

Figure 5. Expression of $\mathrm{MHC}$ genes. $\mathrm{MHCl}(\mathbf{a}), \mathrm{MHC}$ neonatal (b), MHC Ila (c), and MHC IIx (d) mRNA levels in control $(n=5)$ and maternal steroid treatment groups of $2(n=6)$ and 14 days $(n=6)$. Values are expressed relative to median of the control group for quantitative reverse-transcriptase PCR analysis $* P<0.05$.

induce preterm labor in pregnant ewes, and the dose used for pregnant women is a subject of continuing debate. Our current experimental betamethasone dosing strategy does not induce preterm labor in sheep, but it is similar to the minimum dose required to produce lung maturation $(3,22)$. Thus, in the climate of redefining the optimal dosing strategy for antenatal steroids, our finding that a 50\% clinical dosing strategy has no adverse effect on the fetal diaphragm provides 
Table 3. Diaphragm contractile properties

\begin{tabular}{lccc}
\hline & Control & 2-day LPS & 14-day LPS \\
\hline$n$ & 6 & 7 & 8 \\
$P_{\mathrm{O}}\left(\mathrm{N} / \mathrm{cm}^{2}\right)$ & $9.83 \pm 1.56$ & $11.11 \pm 2.62$ & $11.39 \pm 1.91$ \\
$P_{\mathrm{t}}\left(\mathrm{N} / \mathrm{cm}^{2}\right)$ & $5.97 \pm 1.02$ & $5.52 \pm 1.65$ & $6.09 \pm 1.28$ \\
$\mathrm{TTP}(\mathrm{s})$ & $0.147 \pm 0.010$ & $0.135 \pm 0.022$ & $0.140 \pm 0.020$ \\
$1 / 2 \mathrm{RT}(\mathrm{s})$ & $0.303 \pm 0.022$ & $0.255 \pm 0.039$ & $0.275 \pm 0.019$ \\
Max $\mathrm{d} F / \mathrm{d} T(\mathrm{~g} / \mathrm{s})$ & $544 \pm 132$ & $498 \pm 135$ & $471 \pm 179$ \\
Fatigue index & $0.51 \pm 0.13$ & $0.56 \pm 0.09$ & $0.52 \pm 0.09$ \\
\hline $1 / 2$ RT, half relaxation time; Max $\mathrm{d} / \mathrm{d} T$, maximum rate of twitch force development; \\
$P_{\mathrm{O}}$ maximum specific force; $P_{\mathrm{t}}$ peak twitch force; TTP, time to peak twitch force. \\
Values are mean \pm SD.
\end{tabular}

an important support for a lower clinical antenatal betamethasone dosing protocol.

Increased catabolism is a widely reported indicator of glucocorticoid-induced atrophy in adult skeletal muscle $(11,14)$. The expression of the atrophy genes, MuRF1 and $M A F b x$, and UPP, the principal protein degradation pathway, remains unaltered in response to different durations of maternal steroid exposure in our current fetal lamb diaphragm study. The absence of an effect of betamethasone on the proteolytic pathway is consistent with our previous report in the rat pup diaphragm (16). Our data relating to the atrophic response in fetal sheep diaphragm confirm that the antenatal glucocorticoid exposures used in this study had no distinct molecular influence on protein catabolism in the preterm fetal diaphragm.

Muscle atrophy may still arise from depression of the anabolic pathway in the absence of an effect on the catabolic pathway. To explore the protein synthesis signaling cascade, we evaluated four consecutive intracellular components-Akt, mTOR, 4EBP1, and p70S6K. The phosphorylated over total (P/T) Akt content (upstream molecule) was unaltered after a 2- and 14-day low-dose betamethasone exposure used in this study. P/T mTOR protein content appears downregulated by steroid exposure compared with control; however, the absence of significance in the difference between groups $(P=0.102)$ is consistent with the absence of effect of glucocorticoid on the downstream regulation of signaling molecules $\mathrm{p} 70 \mathrm{~S} 6 \mathrm{~K}$ and 4EBP1. In many instances, mTOR coordinately regulates $4 \mathrm{EBP} 1$ and p70S6K (ref. 23). However, under the influence of glucocorticoids, mTOR may differentially regulate p70S6K and 4EBP1 (ref. 24). Our experimental observations show that antenatal betamethasone does not inhibit upstream or downstream mediators of the protein synthesis cascade in fetal lamb diaphragm, which contradicts our previous finding in postnatal rat diaphragm muscle. Multiple factors contribute to these contrasting results including difference in experimental animal species, use of a high dosage in the rat study, and spontaneous breathing of pups from birth until experimentation.

Relatively small sample size compromises the power of this study, and therefore we cannot exclude the possibility of falsenegative errors in some observed results such as P-mTOR

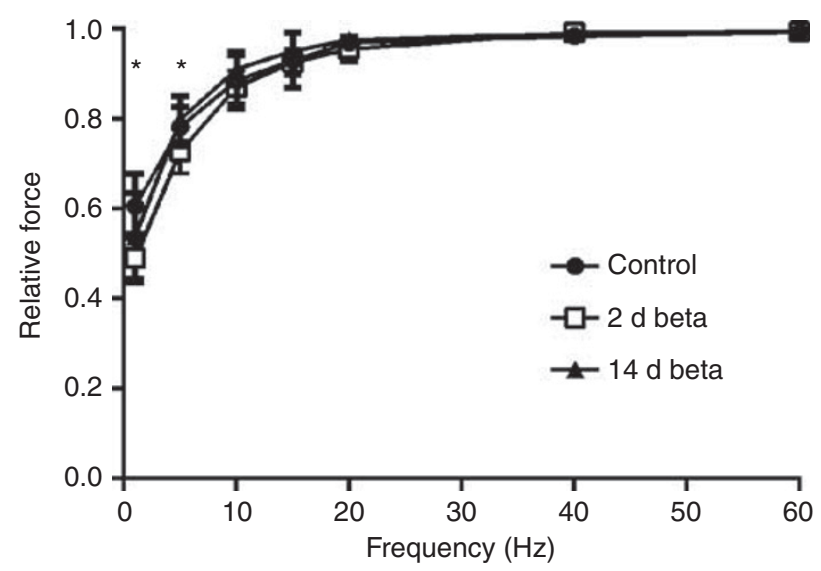

Figure 6. Diaphragm force-frequency relationship. Normalized forcefrequency relationships for control $(n=6)$ and maternal steroid treatment groups of $2(n=7)$ and 14 days $(n=8)$. Values are mean \pm SD. ${ }^{*} P<0.05$.

protein content. However, the absence of a significant change in absolute and relative diaphragm weights indicates the absence of muscle atrophy (loss) after low-dose antenatal betamethasone treatment, which is consistent with the unaltered protein synthesis signaling found in our study.

Decreased protein synthesis is a widely reported cause of adult muscle atrophy in response to glucocorticoid treatment (25). However, the glucocorticoid response varies depending on the gestational age at the time of administration (17). Developmental differences in the effects of steroids may occur because GRs and glucocorticoid-metabolizing enzymes are expressed differentially and regulated by developmental and tissue-specific factors (26,27). Glucocorticoids negatively influence insulin signaling and the associated protein synthesis pathway directly via binding and activating GRs. Subsequently, GRs translocate to the nucleus and bind to genomic glucocorticoid response elements to regulate the transcription of downstream target genes (28). GRs also disrupt insulin signaling through non-genomic mechanisms by increased association with phosphoinositide 3-kinases (PI3K) (29). Our data showed that GR $\alpha$ and its target genes (e.g., IRS1, $p 85 \alpha, k l f 15, I G F 1$, and $I L-10$ ) were not affected at the transcriptional level by maternal steroid exposure, reemphasizing the lack of a negative effect of antenatal glucocorticoid on protein synthesis signaling.

The absence of oxidative stress after antenatal betamethasone exposure in our fetal sheep diaphragms is evident from the indirect experimental measurement of short-lived reactive oxygen species. The antioxidant capacity of the fetus increases with advancing gestation to prepare for the oxygen-rich extrauterine life (7). However, preterm infants have an underdeveloped antioxidant system that makes them more vulnerable to oxidative injury compared with mature infants (30). The transient upregulation of SOD1 gene expression in the 2-day steroid exposure group may be required to counteract increased reactive oxygen species production. Consequently, 


\section{Articles | Mahzabin et al.}

protein carbonylation is not increased by the low-dose antenatal betamethasone applied in our experiment.

The gene expression level of different MHC isoforms were analyzed to examine the effect of antenatal steroids on MHC expression in the preterm diaphragm. Antenatal betamethasone increased MHC II $a$ and IIx gene expression after a 2-day exposure, which did not persist after 14 days. On the other hand, the MHC I mRNA level was not affected by maternal steroid exposure, potentially attributable to lower GR expression in slow-twitch muscle fibers compared with fasttwitch muscle fibers (14). We were unable to report the MHC protein content in maternal steroid-treated fetal diaphragm muscles due to lack of ovine-specific antibodies. Considering the differential expression of MHC gene and proteins in the developing diaphragm $(31,32)$, we speculate that if the gene expression levels translate proportionately into protein levels, we would expect a functional change toward fast contractile characteristics.

We did observe a small reduction in normalized force at low stimulation frequencies after the 2-day betamethasone exposure. This result is consistent with the significant increase in fast type IIa and IIx MHC expression found in the 2-day group, given that the force-stimulation frequency relationship is rightshifted in fast fibers compared with that in slow fibers due to the shorter contraction/relaxation times in fast fibers (33). However, there were no significant changes in twitch contraction and relaxation times or susceptibility to fatigue in steroid-treated fetal diaphragm samples compared with control, although this may be a product of the variability of the nonnormalized data. Ultimately, the small changes in the MHC gene expression and the force-frequency relationship shown here are unlikely to have a detrimental effect on contractile function in the diaphragm, as motor output frequencies in the diaphragm rarely occur below $10 \mathrm{~Hz}$ (refs. 34,35).

These findings suggest that the low-dose maternal betamethasone used in this study does not cause steroid-induced diaphragm weakness when administered 2 or 14 days before delivery. It should be acknowledged that muscle mass was measured without removal of the thin diaphragm ligament. Consequently, cross-sectional area of the diaphragm is likely to be slightly overestimated (see Methods), resulting in a small, consistent underestimation of all specific force values in the present study. This is unlikely to affect the overall interpretation of our results, assuming that steroid exposure did not differentially affect diaphragm ligament mass.

We acknowledge several other study limitations. Clinical translation of ovine studies to human clinical practice is further complicated by differences in doses, gestation periods, developmental trajectories, and potential differential sensitivity to GRs compared with human pregnancy. The effects of glucocorticoids on lung function and growth velocity in humans appear to be smaller than those observed in animal models (36). Further studies are needed to explore whether the early and late postnatal developmental events are influenced by maternal steroid exposure.
Contrary to our hypothesis, our data suggest that low-dose maternal betamethasone administered up to 2 weeks prior to delivery of the fetal lamb at $80 \%$ gestation has no sustained detrimental effect on preterm diaphragm integrity in terms of protein metabolism, cellular oxidative status, MHC fibre-type composition, and functional properties. Our findings are consistent with the positive influence of antenatal betamethasone on fetal lung maturation established from clinical trials and animal studies. There is minimal evidence of adverse diaphragm function for the offspring following a single course of low-dose antenatal betamethasone. Thus, our preclinical research further supports the use of low-dose antenatal glucocorticoid in the clinical settings for maximal respiratory benefits.

\section{ACKNOWLEDGMENTS}

The study was supported by a National Health and Medical Research Council (NHMRC) Centre for Research Excellence (1057514), NHMRC Project Grant (1010665), NHMRC Senior Research Fellowship (1077691) and Career Development Fellowship (1045824) and the Women and Infants Research Foundation.

Disclosure: The authors declare no conflict of interest.

\section{REFERENCES}

1. Leviton LC, Goldenberg RL, Baker CS, et al. Methods to encourage the use of antenatal corticosteroid therapy for fetal maturation - A randomized controlled trial. J Am Med Assoc 1999;281:46-52.

2. Bonanno C, Fuchs K, Wapner RJ. Single versus repeat courses of antenatal steroids to improve neonatal outcomes: risks and benefits. Obstet Gynecol Surv 2007;62:261-71.

3. Kemp MW, Saito M, Usuda H, et al. Materno-fetal pharmacokinetics and fetal lung responses in chronically catheterised sheep receiving constant, low-dose infusions of betamethasone phosphate. Am J Obstet Gynecol 2016;215:775, e1-12.

4. Keens TG, Bryan AC, Levison H, Ianuzzo CD. Developmental pattern of muscle in human ventilatory muscles fiber types. J Appl Physiol 1978;44: 909-13.

5. West JM, Barclay CJ, Luff AR, Walker DW. Developmental changes in the activation properties and ultrastructure of fast- and slow-twitch muscles from fetal sheep. J Muscle Res Cell Motil 1999;20:249-64.

6. Lavin T, Song Y, Bakker AJ, et al. Developmental changes in diaphragm muscle function in the preterm and postnatal lamb. Pediatr Pulmonol 2013;48:640-8.

7. Song Y, Pillow JJ. Ontogeny of proteolytic signaling and antioxidant capacity in fetal and neonatal diaphragm. Anat Rec 2012;295:864-71.

8. Song Y, Pillow JJ. Developmental regulation of molecular signalling in fetal and neonatal diaphragm protein metabolism. Exp Biol Med 2013;238:913-22.

9. Dekhuijzen PNR, Decramer M. Steroid-induced myopathy and its significance to respiratory disease: A known disease rediscovered. Eur Respir J 1992;5:997-1003.

10. Sartorelli V, Fulco M. Molecular and cellular determinants of skeletal muscle atrophy and hypertrophy. Sci STKE 2004;2004:re11.

11. Bonaldo P, Sandri M. Cellular and molecular mechanisms of muscle atrophy. Dis Model Mech 2013;6:25-39.

12. Schakman O, Gilson H, Thissen JP. Mechanisms of glucocorticoidinduced myopathy. J Endocrinol 2008;197:1-10.

13. Eason JM, Dodd SL, Powers SK, Martin AD. Detrimental effects of shortterm glucocorticoid use on the rat diaphragm. Phys Ther 2000;80:160-7.

14. Schakman O, Kalista S, Barbé C, Loumaye A, Thissen JP. Glucocorticoidinduced skeletal muscle atrophy. Int J Biochem Cell Biol 2013;45: 2163-72. 
15. Viguerie N, Picard F, Hul G, et al. Multiple effects of a short-term dexamethasone treatment in human skeletal muscle and adipose tissue. Physiol Genomics 2012;44:141-51.

16. Song Y, Demmer DL, Pinniger GJ, et al. Effect of maternal steroid on developing diaphragm integrity. PLoS One 2014;9:e93224.

17. Blanco CL, Moreira AG, McGill LL, Anzueto DG, Nathanielsz P, Musi N. Antenatal corticosteroids alter insulin signaling pathways in fetal baboon skeletal muscle. J Endocrinol 2014;221:253-60.

18. Jellyman JK, Martin-Gronert MS, Cripps RL, et al. Effects of cortisol and dexamethasone on insulin signalling pathways in skeletal muscle of the ovine fetus during late gestation. PLoS ONE 2012;7:1-7.

19. Livak KJ, Schmittgen TD. Analysis of relative gene expression data using real-time quantitative PCR and the 2(-Delta Delta $C(T)$ ) Method. Methods 2001;25:402-8.

20. Javen I, Williams NA, Young IR, Luff AR, Walker D. Growth and differentiation of fast and slow muscles in fetal sheep, and the effects of hypophysectomy. J Physiol 1996;494:839-49.

21. Liggins GC, Howie RN. A controlled trial of antepartum glucoccorticoid treatment for prevention of the respiratory distress syndrome in premature infants. Pediatrics 1972;50:515-25.

22. Loehle M, Schwab M, Kadner S, et al. Dose-response effects of betamethasone on maturation of the fetal sheep lung. Am J Obstet Gynecol 2010;125:2621-9.

23. Hara K, Yonezawa K, Weng QP, Kozlowski MT, Belham CAJ. Amino acid sufficiency and mTOR regulate p70 S6 kinase and eIF-4E BP1 through a common effector mechanism. J Biol Chem 1998;273: 14484-94.

24. Shah OJ, Kimball SR, Jefferson LS. Among translational effectors, p70S6k is uniquely sensitive to inhibition by glucocorticoids. Biochem J 2000;347 (Pt 2): 389-97.

25. Savary I, Debras E, Dardevet D, et al. Effect of glucocorticoid excess on skeletal muscle and heart protein synthesis in adult and old rats. Br J Nutr 1998;79:297-304.
26. Speirs HJL, Seckl JR, Brown RW. Ontogeny of glucocorticoid receptor and 11beta-hydroxysteroid dehydrogenase type-1 gene expression identifies potential critical periods of glucocorticoid susceptibility during development. J Endocrinol 2004;181:105-6.

27. Kalinyak JE, Griffin CA, Hamilton RW, Bradshaw JG, Perlman AJ, Hoffman AR. Developmental and hormonal regulation of glucocorticoid receptor messenger RNA in the rat. J Clin Invest 1989;84:1843-8.

28. Kuo T, Lew MJ, Mayba O, Harris CA., Speed TP, Wang JC. Genome-wide analysis of glucocorticoid receptor-binding sites in myotubes identifies gene networks modulating insulin signaling. Proc Natl Acad Sci USA 2012;109:11160-5.

29. Hu Z, Wang H, In HL, Du J, Mitch WE. Endogenous glucocorticoids and impaired insulin signaling are both required to stimulate muscle wasting under pathophysiological conditions in mice. J Clin Invest 2009;119: 3059-69.

30. Asikainen TM, Raivio KO, Saksela M. Expression and developmental profile of antioxidant enzymes in human lung and liver. Am J Respir Cell Mol Biol 1998;19:942-9.

31. Geiger PC, Bailey JP, Mantilla CB, Zhan WZ, Sieck GC. Mechanisms underlying myosin heavy chain expression during development of the rat diaphragm muscle. J Appl Physiol 2006;101:1546-55.

32. Eizema K, van den Burg M, Kiri A, et al. Differential expression of equine myosin heavy-chain mRNA and protein isoforms in a limb muscle. J Histochem Cytochem 2003;51:1207-6.

33. Close RI.. Dynamic mammalian properties of skeletal muscles. Physiol Rev 1972;52:129-97.

34. Kowalski KE, Hsieh YH, Dick TE, DiMarco AF. Diaphragm activation via high frequency spinal cord stimulation in a rodent model of spinal cord injury. Exp Neurol 2013;247:689-93.

35. Butler JE, Hudson AL, Gandevia SC. The neural control of human inspiratory muscles. Prog Brain Res 2014;209:295-308.

36. Newnham JP, Jobe AH. Should we be prescribing repeated courses of antenatal corticosteroids? Semin Fetal Neonatal Med 2009;14:157-63. 